\title{
EVALUATION OF JOINT FLEXIBILITY AND CARDIOVASCULAR EFFICIENCY IN CHILDREN AND ADOLESCENTS WITH HAEMOPHILIA AND THEIR HEALTHY PEERS
}

\author{
Eva Mihalova
}

Department of Paediatrics, Faculty of Medicine and Dentistry, Palacký University Olomouc, Czech Republic e-mail:Eva.Mihalova@seznam.cz

Received: September 29, 2006; Accepted (with revision): March 21, 2007

Key words: Haemophilia/Physiotherapy/Step test/Goniometry

Background: Problems concerning treatment of patients with haemophilia are long-term and exist even in the present days. Thanks to interdisciplinary complex therapy the results of treatment are constantly better than many years ago.

Aim: The goal of this study is to analyse the current state of management of haemophilia and to suggest a comprehensive concept of rational and effective rehabilitation of children with haemophilia.

Method: In the clinical study, two different measurements were used (goniometry and functional step test) and a special questionnaire. Four different groups of participants were created for the clinical measurements (a set of patients and a control group) and for the questionnaire (a set of patients and a control group).

Results: This study concluded that there were no significant differences in the outcomes of the goniometry and the step test, and in the restriction of movement activities between the patients and healthy individuals.

Conclusions: The concept of physiotherapy in patients with coagulopathies in the Czech Republic is comparable with rehabilitation concepts in other European and non-European countries. Current rehabilitation care is of good quality and necessary for effective lifelong therapy of haemophilia, however, it is used insufficiently.

\section{INTRODUCTION}

Over the last forty years, treatment of haemophilia has reached a high level of quality. Thanks to doctors, physical therapists, psychologists, social workers and other professionals it is much easier to minimize permanent consequences of the disease. Issues concerning the efficacy of complex treatment of haemophilia (substitution, prophylaxis, home therapy, physical therapy etc.) and their may be encountered in many scientific articles. It is evident that physical therapy is an indispensable and important component of care for patients with coagulation disorders. Despite the fact that good-quality rehabilitation is an integral part of effective long-term treatment of the disease it is only used in few cases. This is due to a lack of specialized rehabilitation centres, their accessibility, finance and, above all, motivation of patients.

The goal of the study was to evaluate joint flexibility using goniometry and cardiovascular efficiency by a functional step test in children and adolescent with haemophilia and von Willebrand's disease as well as to compare the values with those of their healthy peers. The questionnaire was supposed to show, among others, the attitude of patients and healthy individuals to physical activities. We focused on improving the physical state of patients with haemophilia A or B and patients with von Willebrand's disease. We also tried to compare the questionnaire, go- niometry, and functional step test results of the patients and relevant control groups ${ }^{3}$.

\section{MATERIAL AND METHODS}

The group for clinical measurements (goniometry, step test) comprised 13 patients aged 13 to 25 years with haemophilia A or B (severe, moderate or mild forms) and patients with von Willebrand's disease (a severe form), treated in the Children's Medical Centre in Brno and the Department of Paediatrics of the University Hospital in Olomouc. With respect to their health condition, all patients were able to complete the clinical study. In the clinical measurement control group, there were 13 healthy individuals in the same age range as the patients. The questionnaires were used in 29 patients aged 8-25 suffering from haemophilia A or B and or from von Willebrand's disease who were treated in the aforementioned facilities in Brno and Olomouc. The relevant control group consisted of 29 healthy subjects of the same age range ${ }^{3}$.

All subjects were acquainted with our requirements which included filling in the questionnaire and participation in the clinical measurements in specified times. The measurements took the place in the Children's Medical Centre in Brno and/or the Department of Paediatrics or the Department of Sports and Exercise Medicine of the 
Table 1. The results of goniometry measurement of lower limbs (according to the SFTR method).

\begin{tabular}{|c|c|c|c|}
\hline \multicolumn{4}{|c|}{ GONIOMETRY MEASUREMENT } \\
\hline & Patients & Control subjects & Comparison \\
\hline \multicolumn{4}{|l|}{ Ankle $\mathrm{S}$} \\
\hline DF: $1 . \sin$. & $20.77^{\circ}$ & $13.46^{\circ}$ & no significant difference $(\mathrm{p}=0.22)$ \\
\hline DF: $1 . d x$ & $20^{\circ}$ & $14.23^{\circ}$ & no significant difference $(\mathrm{p}=0.34)$ \\
\hline PF: 1. sin. & $35^{\circ}$ & $47.69^{\circ}$ & significant difference $(p=0.02)$ \\
\hline PF: 1. dx. & $34.61^{\circ}$ & $48.46^{\circ}$ & significant difference $(p=0.02)$ \\
\hline \multicolumn{4}{|l|}{ knee S } \\
\hline F: $1 . \sin$. & $111.54^{\circ}$ & $118.46^{\circ}$ & no significant difference $(p=0.51)$ \\
\hline F: 1. dx. & $109.23^{\circ}$ & $120.77^{\circ}$ & no significant difference $(p=0.28)$ \\
\hline \multicolumn{4}{|l|}{ hip S/F/R } \\
\hline E: 1. sin. & $16.15^{\circ}$ & $20.38^{\circ}$ & significant difference $(p=0.007)$ \\
\hline E: 1. dx. & $17.69^{\circ}$ & $20.77^{\circ}$ & no significant difference $(p=0.07)$ \\
\hline $\mathrm{F}: 1 . \sin$ & $89.23^{\circ}$ & $80^{\circ}$ & no significant difference $(\mathrm{p}=0.23)$ \\
\hline F: 1. dx. & $90.38^{\circ}$ & $81.15^{\circ}$ & no significant difference $(p=0.22)$ \\
\hline $\mathrm{AB}: 1 . \sin$. & $32.31^{\circ}$ & $35.38^{\circ}$ & no significant difference $(p=0.20)$ \\
\hline$A B: 1 . d x$. & $32.31^{\circ}$ & $34.23^{\circ}$ & no significant difference $(p=0.44)$ \\
\hline AD: 1. sin. & $20.38^{\circ}$ & $22.30^{\circ}$ & no significant difference $(p=0.55)$ \\
\hline AD: $1 . d x$ & $19.62^{\circ}$ & $23.08^{\circ}$ & no significant difference $(p=0.24)$ \\
\hline ER: 1. sin. & $43.08^{\circ}$ & $39.23^{\circ}$ & no significant difference $(p=0.15)$ \\
\hline ER: 1. dx. & $41.15^{\circ}$ & $40.77^{\circ}$ & no significant difference $(\mathrm{p}=0.85)$ \\
\hline IR: 1. sin. & $42.69^{\circ}$ & $34.23^{\circ}$ & significant difference $(p=0.02)$ \\
\hline IR: 1. dx. & $42.69^{\circ}$ & $36.15^{\circ}$ & significant difference $(\mathrm{p}=0.007)$ \\
\hline
\end{tabular}

$\mathrm{DF}=$ dorsal flexion, $\mathrm{PF}=$ plantar flexion, $\mathrm{F}=$ flexion, $\mathrm{E}=$ extension, $\mathrm{AB}=$ abduction, $\mathrm{AD}=$ adduction, $\mathrm{ER}=$ external rotation, $\mathrm{IR}=$ internal rotation, 1 . $\sin .=$ left side, $1 . \mathrm{dx} .=$ right side.

Table 2. The functional step test results.

\begin{tabular}{|l|l|l|}
\hline \multicolumn{3}{|c|}{ STEP TEST } \\
\hline & Patients & Control subjects \\
\hline Initial pulse & $86.44 \mathrm{pulse} / \mathrm{min}$ & $94.23 \mathrm{pulse} / \mathrm{min}$ \\
Pulse in minute 1 & $95.46 \mathrm{pulse} / \mathrm{min}$ & $92.85 \mathrm{pulse} / \mathrm{min}$ \\
Pulse in minute 2 & $90.92 \mathrm{pulse} / \mathrm{min}$ & $88.46 \mathrm{pulse} / \mathrm{min}$ \\
Pulse in minute 3 & $86.54 \mathrm{pulse} / \mathrm{min}$ & $86.69 \mathrm{pulse} / \mathrm{min}$ \\
Fitness index & 57.46 & 57.23 \\
\hline
\end{tabular}


University Hospital in Olomouc. We prepared two versions of the questionnaire concerning physical activities, leisure time and lifestyle - one for the patients and the other one for the controls. The questionnaires were the same but the patients' one contained four extra questions concerning illness ${ }^{3}$.

The clinical measurements were performed in a study room with an examination table. Apart from the patient and the investigator, sometimes the patient's parents were present in the room. The measurements included the patient's history, goniometric assessment of joint range of motion (apart from small joints of the hand and foot) using the SFTR method ( $\mathrm{S}=$ sagital plane, $\mathrm{F}$ = frontal plane, $\mathrm{T}=$ transversal plane, $\mathrm{R}=$ rotation) and the step test. During the step test, the pulse frequency was measured with the Polar Sport Tester in regular time intervals and then the fitness index was calculated from the obtained values: fitness index $=($ exercise length in sec. $x 100) /$ (count of 3 pulse periods $x 2$ ). According to the fitness index, we could grade the subjects' physical condition $(\mathrm{PhC})$. Low $\mathrm{PhC}$ is below 50 points of the fitness index, low-average $\mathrm{PhC}$ between 55 and 64 points, high-average $\mathrm{PhC}$ between 65 and 79 points, efficiency - from 80 to 89 points and high efficiency less than 90 points of the fitness index. The Czech modification of the Harvard Step Test was used. The goniometry and step test results were statistically processed by two-selective independent T-test with the level of statistical significance $p=0.05$. The resultant values from the questionnaires were evaluated by percentage $^{3}$.

\section{RESULTS AND DISCUSSION}

The study revealed no significant differences in the goniometry and step test outcomes or in restriction of movement activities between patients and healthy individuals. (Tab. 1, 2) Another important fact is that the concept of physiotherapy in patients with coagulopathies in the Czech Republic is comparable with rehabilitation concepts in other European and non-European countries ${ }^{3}$.

In 2001, Schoenmakers and colleagues studied 39 children aged from 4 to 12 with moderate and severe haemophilia to find out whether haemophilic children on prophylactic therapy differed from their healthy peers in terms of motor performance and disability. They assessed range of joint motion, muscle strength, motor control and ADL (activities of daily living). The normal range of motion in all joints was seen in $97 \%$ (38/39) of the patients. Strength of elbow, knee and ankle muscles were within the normal ranges. Ninety-five percent (37/39) of the patients had normal motor performance. Although $90 \%$ of their patients $(35 / 39)$ had no disabilities in ADL, restrictions in sports or gymnastics were seen in $56 \%(22 / 39)$ of the patients. The Dutch researchers also found no signifi- cant differences between the patients and healthy control group in motor manifestation and $\mathrm{ADL}^{5}$.

Our questionnaire was focused on the physical, psychological and social aspects. The physical aspect questions dealt with the patients' and healthy subjects' scope of indoor/outdoor sport activities, restrictions in sport (if any), daily amounts of time spent watching TV/video/DVD or working on a PC etc. In the psychological and social areas, we were interested in their relationships with parents, siblings, healthy peers etc. In most cases, there were only minimal differences between the answers of the patients and their healthy peers. Most of them mentioned some regular indoor/outdoor sport activities: $68.97 \%(20 / 29)$ of the patients and $86.21 \%(25 / 29)$ of the healthy individuals. As many as $31.03 \%$ (9/29) of the patients and $20.69 \%(6 / 29)$ of the controls exercise daily. Quite surprisingly, only $20.69 \%(6 / 29)$ of the patients regularly attended rehabilitation therapy. Physiotherapy should be an integral part of comprehensive treatment of haemophilia, not only during hospitalization but especially during home therapy.

The results of the step test displayed no significant differences in physical condition between the patients and the healthy controls. Unfortunately, both groups fell within the low average range of physical condition (Tab. 2), presumably due to their lifestyle.

The study revealed many interesting yet unsettling facts. Despite the high quality of current rehabilitation care necessary for effective lifelong therapy of haemophilia it is used rather insufficiently. This fact is influenced by a lack of specialized facilities and their availability, finance and motivation of patients as well as their parents and physiotherapists. It is essential to involve parents in the therapy to prepare them for the subsequent home care ${ }^{1,4}$. For example, a Canadian pilot study assessed a physiotherapeutic home care programme focused especially on proprioceptive and balance exercises. Its main goal was to reduce the number of traumatic bleeding in the ankles ${ }^{2}$.

\section{REFERENCES}

1. Buzzard BM. Proprioceptive training in haemophilia. Haemophilia 1998; 4:528-531.

2. Various authors. Physiotherapy. Haemophilia 2002; 8:482-486.

3. Mihálová E. Problematika léčebné rehabilitace u hemofiliků. Thesis. 2006.

4. Santavirta N, Solovieva S, Helkama O, Lehto S, Konttinen YT, Santavirta S. Musculoskeletal pain and functional ability in haemophilia A and B. Physiotherapy and rehabilitation in haemophilia patient. Rheumatology International. 2001; http://springerlink. metapress.com/app/home/contribution.asp.

5. Schoenmakers MAGC, Gulmans VAM, Helders PJM, Van Den Berg HM. Motor performance and disability in Dutch children with haemophilia: a comparison with their healthy peers. Haemophilia 2001; 7:293-298. 